\title{
Accurate Approximation of Eigenvalues and Zeros of Selected Eigenfunctions of Regular Sturm-Liouville Problems
}

\author{
By Eugene C. Gartland, Jr.
}

\begin{abstract}
A method for simultaneously approximating to high accuracy the corresponding eigenvalue and zeros of the $(n+1)$ st eigenfunction of a regular Sturm-Liouville eigenvalue problem is presented. It is based upon equilibrating the minimum eigenvalues of several problems on subintervals that form a partition of the orginal interval. The method is easily derived from classical mini-max variational principles. The equilibration is accomplished iteratively using an approximate Newton Method. Numerical results are given.
\end{abstract}

Introduction. The problem of approximating the eigenvalues of regular SturmLiouville equations has attracted the attention of analysts for a long time. An annoying aspect of most numerical schemes is that the accuracy of the approximation of the $n$th eigenvalue decreases as $n$ increases. This is due to the fact that the higher eigenfunctions are more oscillatory and therefore more difficult to approximate accurately.

In this paper is presented a simple method by which any eigenvalue can be approximated as accurately as the first, which accuracy will depend of course on the particular scheme utilized for this basic (minimum eigenvalue) calculation. The method is based upon approximating the minimum eigenvalues of several problems on subintervals that form a partition of the original interval. These "subeigenvalues" are then equilibrated by iteratively selecting appropriate breakpoints for the subintervals.

A related question, for Sturm-Liouville problems, is that of calculating the $n$ distinct zeros of the $(n+1)$ st eigenfunction. These points are of interest in some applications. One approach to approximating the zeros of such a "special function" would be to use a standard root finder together with an analytic approximation (such as a truncated series or continued fraction) to the eigenfunction. The accuracy of the computed zeros then would depend on the accuracy of the approximation to the eigenfunction.

Here we will see that these zeros are precisely the equilibration points mentioned previously. Thus they can be approximated, simultaneously with the eigenvalue, in a general way, which requires no specific information about the eigenfunction; and the accuracy of the approximation will depend only on the accuracy to which one can approximate the minimum eigenvalues on the subproblems.

Received August 30, 1982.

1980 Mathematics Subject Classification. Primary 65L15.

Key words and phrases. Eigenvalues, zeros of eigenfunctions, Sturm-Liouville problems. 
The theoretical material consists mostly of simple observations based on familiar mini-max principles and is contained in the next two sections. The iterative scheme that solves the equilibration problem is an approximate Newton method and is developed in Section 3. Numerical results are given in Section 4.

1. The Problem. Consider the regular Sturm-Liouville eigenvalue problem

$$
\begin{gathered}
L \phi \equiv-\left(p \phi^{\prime}\right)^{\prime}+q \phi=\Lambda s \phi, \quad 0<x<1, \\
\phi(0)=\phi(1)=0{ }^{*}
\end{gathered}
$$

It is classical (see [2]) that if the real functions $p, q$, and $s$ are sufficiently smooth with $p$ and $s$ positive then (1.1) possesses an infinite sequence of real, simple eigenvalues $-\infty<\Lambda_{1}<\Lambda_{2}<\cdots$ (with no finite accumulation point) and corresponding eigenfunctions $\Phi_{1}, \Phi_{2}, \ldots$ satisfying $\left(\Phi_{i}, \Phi_{j}\right)_{s}=\delta_{i j}, i, j=1,2, \ldots$ Here $(\cdot, \cdot)_{s}$ denotes the inner product on the weighted $L^{2}$ space $L^{2}([0,1], s)$ :

$$
(\phi, \psi)_{s} \equiv \int_{0}^{1} \phi \psi s .
$$

The $(n+1)$ st eigenfunction, $\Phi_{n+1}$, has exactly $n$ distinct zeros $0<\xi_{1}<\cdots<\xi_{n}<$ 1 in $(0,1)$.

With the differential operator $L$ and the boundary conditions of (1.1) we can associate the bilinear form

$$
a(\phi, \psi) \equiv \int_{0}^{1}\left(p \phi^{\prime} \psi^{\prime}+q \phi \psi\right)
$$

Let $(\cdot, \cdot)$ denote the usual $L^{2}[0,1]$ inner product. Then the form above results from integration by parts of $(L \phi, \psi)$ for sufficiently smooth $\phi$ and $\psi$ vanishing at 0 and 1 , and for such functions we have $(L \phi, \psi)=a(\phi, \psi)=(\phi, L \psi)$.

The eigenvalues of (1.1) are stationary values of the Rayleigh quotient $a(\phi, \phi) /(\phi, \phi)_{s}$. In fact, we have (see [7]) the variational characterization

$$
\Lambda_{n+1}=\sup _{\substack{l_{1} \ldots \ldots l_{n} \\ \text { linear functionals }}} l_{1(\phi)=\cdots=l_{n}(\phi)=0} \frac{a(\phi, \phi)}{(\phi, \phi)_{s}} .
$$

Here and in the sequel, admissible functions are sufficiently smooth (continuous with piecewise continuous derivative), nontrivial functions that vanish at 0 and 1 . The extremum above is achieved by the $(n+1)$ st eigenfunction, $\Phi_{n+1}$, when the linear functionals are given by

$$
l_{i}(\phi)=\left(\phi, \Phi_{i}\right)_{s}, \quad i=1, \ldots, n .
$$

We are interested in approximating to high accuracy the eigenvalue $\Lambda_{n+1}$ and the zeros $\xi_{1}, \ldots, \xi_{n}$ of $\Phi_{n+1}$. We will find a slightly different variational characterization helpful.

2. Connection With an Equilibration Problem. The linear functionals (1.3), for which the supremum in (1.2) is attained, are not unique. It so happens that the supremum is also attained at the functionals of point evaluation at the zeros of $\Phi_{n+1}$. This can be deduced as follows.

*More general separated boundary conditions of the form $\alpha_{0} \phi(0)-\beta_{0} p \phi^{\prime}(0)=0, \alpha_{1} \phi(1)+\beta_{1} p \phi^{\prime}(1)$ $=0$, as well as an arbitrary finite interval, can be handled similarly. The problem (1.1) is chosen for definiteness and simplicity. 
Let $D$ denote the open set $\left\{\mathbf{x}=\left(x_{1}, \ldots, x_{n}\right): 0<x_{1}<\cdots<x_{n}<1\right\}$ in $\mathbf{R}^{n}$. As convention take $x_{0}=0$ and $x_{n+1}=1$. For any $\mathbf{x}$ in $D$ define

$$
a(\phi, \psi)_{i} \equiv \int_{x_{i}}^{x_{i+1}}\left(p \phi^{\prime} \psi^{\prime}+q \phi \psi\right)
$$

and

$$
(\phi, \psi)_{s, i} \equiv \int_{x_{i}}^{x_{i+1}} \phi \psi s, \quad i=0, \ldots, n
$$

Let $\lambda_{i}$ denote the minimum eigenvalue of the differential operator $L$ on the subinterval $\left[x_{i}, x_{i+1}\right]$ with zero endpoint conditions. Then $\lambda_{i}$ is a function of $\mathbf{x}$, and

$$
\lambda_{i}=\inf _{\phi\left(x_{i}\right)=\phi\left(x_{i+1}\right)=0} \frac{a(\phi, \phi)_{i}}{(\phi, \phi)_{s, i}}, \quad i=0, \ldots, n .
$$

Proposition 2.1. Let $\mathbf{x}$ be in $D, \lambda_{0}, \ldots, \lambda_{n}$ be as above, and $\lambda_{\min }(\mathbf{x})$ denote $\min \left\{\lambda_{0}, \ldots, \lambda_{n}\right\}$. Then

$$
\inf _{\phi\left(x_{1}\right)=\cdots=\phi\left(x_{n}\right)=0} \frac{a(\phi, \phi)}{(\phi, \phi)_{s}}=\lambda_{\min }(\mathbf{x}) .
$$

Proof. If $\phi$ is sufficiently smooth and vanishes at $x_{1}, \ldots, x_{n}$ (as well as at 0 and 1 ), then

$$
\frac{a(\phi, \phi)}{(\phi, \phi)_{s}}=\frac{\Sigma a(\phi, \phi)_{i}}{\Sigma(\phi, \phi)_{s, i}} \geqslant \frac{\Sigma \lambda_{i}(\phi, \phi)_{s, i}}{\Sigma(\phi, \phi)_{s, i}} \geqslant \lambda_{\min }(\mathbf{x})
$$

Equality is attained when $\phi$ is an eigenfunction associated with the eigenvalue $\lambda_{\min }(\mathbf{x})$ and extended zero outside an interval corresponding to this minimum eigenvalue.

In the case where the points $x_{1}, \ldots, x_{n}$ are located at the zeros $\xi_{1}, \ldots, \xi_{n}$ of the normalized eigenfunction $\Phi_{n+1}$, we get

$$
\Lambda_{n+1}=a\left(\Phi_{n+1}, \Phi_{n+1}\right)=\frac{a\left(\Phi_{n+1}, \Phi_{n+1}\right)_{i}}{\left(\Phi_{n+1}, \Phi_{n+1}\right)_{s, i}}, \quad i=0, \ldots, n .
$$

The second equality simply follows by integration by parts and use of the facts that $L \Phi_{n+1}=\Lambda_{n+1} s \Phi_{n+1}$ and $\Phi_{n+1}\left(x_{i}\right)=\Phi_{n+1}\left(x_{i+1}\right)=0$ (since $x_{i}=\xi_{i}$ and $x_{i+1}=$ $\left.\xi_{i+1}\right)$. The restriction of $\Phi_{n+1}$ to the interval $\left[x_{i}, x_{i+1}\right]$ is an eigenfunction corresponding to the minimum eigenvalue $\lambda_{i}$ on this subinterval, and $\lambda_{i}=\Lambda_{n+1}$. Therefore $\lambda_{\min }(\xi)=\Lambda_{n+1}$, and we have the following

Proposition 2.2. The $(n+1)$ st eigenvalue, $\Lambda_{n+1}$, satisfies

$$
\Lambda_{n+1}=\sup _{0<x_{1}<\cdots<x_{n}<1} \inf _{\phi\left(x_{1}\right)=\cdots=\phi\left(x_{n}\right)=0} \frac{a(\phi, \phi)}{(\phi, \phi)_{s}},
$$

and the extremum is achieved by the $(n+1)$ st eigenfunction, $\Phi_{n+1}$, when $x_{1}, \ldots, x_{n}$ are taken to be the zeros of this function. 
Proof. From Proposition 2.1 and the observations above we have

$$
\begin{aligned}
\Lambda_{n+1} & =\inf _{\phi\left(\xi_{1}\right)=\cdots=\phi\left(\xi_{n}\right)=0} \frac{a(\phi, \phi)}{(\phi, \phi)_{s}} \\
& \leqslant \sup _{0<x_{1}<\cdots<x_{n}<1} \quad \inf _{\phi\left(x_{1}\right)=\cdots=\phi\left(x_{n}\right)=0} \frac{a(\phi, \phi)}{(\phi, \phi)_{s}} \\
& \leqslant \sup _{\substack{l_{1}, \ldots, l_{n} \\
\text { linear functionals }}} l_{1}(\phi)=\cdots=l_{n}(\phi)=0
\end{aligned}
$$

The "subeigenvalues" $\lambda_{0}, \ldots, \lambda_{n}$ (the minimum eigenvalues of the problems on the subintervals) are continuous, strictly decreasing functions of the subintervals, that is, if $\left[x_{i}, x_{i+1}\right]$ is properly contained in $\left[x_{i}^{\prime}, x_{i+1}^{\prime}\right]$, then $\lambda_{i}>\lambda_{i}^{\prime}$ and $\lambda_{i}^{\prime} \rightarrow \lambda_{i}$ as $\|\left[x_{i}^{\prime}, x_{i+1}^{\prime}\right] \backslash\left[x_{i}, x_{i+1}\right] \rightarrow 0$. It follows that the set of points $\mathbf{x}=\left(x_{1}, \ldots, x_{n}\right)$ that maximizes $\lambda_{\min }(\mathbf{x})$ must be such that the subeigenvalues are equilibrated: if these were not equilibrated, then one could increase $\lambda_{\min }(\mathbf{x})$ by shrinking those subintervals corresponding to this minimum value. It also follows that this set of points is unique: any other point set must be such that it possesses a subinterval that properly contains a subinterval of the optimal set, and it must therefore have a strictly smaller $\lambda_{\min }(\mathbf{x})$. As already observed, these points must be the zeros of $\Phi_{n+1}$. Moreover, for any $\mathbf{x}$ in $D$ we have $\lambda_{\text {min }}(\mathbf{x}) \leqslant \Lambda_{n+1} \leqslant \lambda_{\max }(\mathbf{x})$, where $\lambda_{\text {min }}$ and $\lambda_{\max }$ denote the minimum and maximum of the subeigenvalues $\lambda_{0}, \ldots, \lambda_{n}$ on the partition induced by $\mathbf{x}$. These results are summarized, for later reference, in the theorem below.

THEOREM 2.3. The zeros of the $(n+1)$ st eigenfunction provide the unique maximizing points for the supremum in (2.1). They are uniquely determined by the equilibration condition

$$
\lambda_{0}(\mathbf{x})=\cdots=\lambda_{n}(\mathbf{x})
$$

Furthermore, for any $\mathbf{x}$ in $D$ we have

$$
\min \left\{\lambda_{0}(\mathbf{x}), \ldots, \lambda_{n}(\mathbf{x})\right\} \leqslant \Lambda_{n+1} \leqslant \max \left\{\lambda_{0}(\mathbf{x}), \ldots, \lambda_{n}(\mathbf{x})\right\}
$$

These observations provide us with a way of approximating the zeros of $\Phi_{n+1}$ by equilibrating the minimum eigenvalues of subproblems, producing brackettings for $\Lambda_{n+1}$ along the way. The only limitation on the attainable accuracy of the zeros or the eigenvalue is the accuracy to which one is able to approximate the first eigenvalues on the subintervals. Thus, in theory, we can approximate any finite number of eigenvalues to the same order of accuracy and not have to accept the usual deterioration in the accuracy of the higher values. The tradeoff is in the number of minimum eigenvalues that must be approximated. To make the approach at all practical, an efficient scheme to equilibrate $\lambda_{0}(\mathbf{x}), \ldots, \lambda_{n}(\mathbf{x})$ is needed. For this, Newton's method can be employed. 
3. Newton's Method for the Equilibration Problem. The equilibration condition of Theorem 2.3, which uniquely determines the zeros $\xi_{1}, \ldots, \xi_{n}$, can be written as a vector equation $F(\mathbf{x})=0$ with

$$
F(\mathbf{x})=\left[\begin{array}{c}
F_{1}(\mathbf{x}) \\
F_{2}(\mathbf{x}) \\
\vdots \\
F_{n}(\mathbf{x})
\end{array}\right] \equiv\left[\begin{array}{c}
\lambda_{0}(\mathbf{x})-\lambda_{1}(\mathbf{x}) \\
\lambda_{1}(\mathbf{x})-\lambda_{2}(\mathbf{x}) \\
\vdots \\
\lambda_{n-1}(\mathbf{x})-\lambda_{n}(\mathbf{x})
\end{array}\right]
$$

Actually, $\lambda_{i}$ depends only on $x_{i}$ and $x_{i+1}$; so the Jacobian $F^{\prime}(\mathbf{x})$ is tridiagonal and has the form

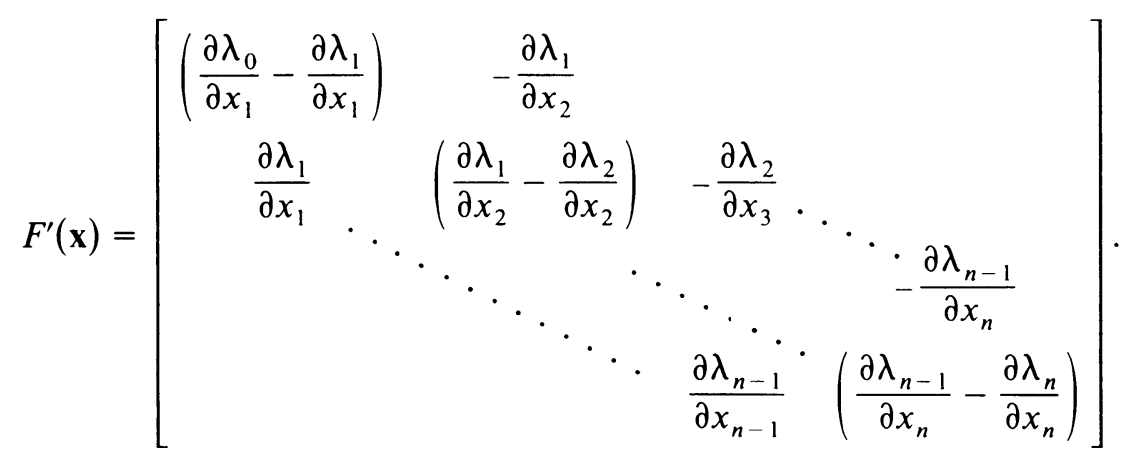

To use Newton's method, then, we require some information about the partial derivatives of the subeigenvalues with respect to the endpoints of the subintervals.

The theory of perturbations of a linear operator can be used to conclude that under appropriate conditions on the coefficients $p, q$, and $s$ these partial derivatives exist and are continuous. The standard reference here is [3]. Our problem can be put into this context by taking a perturbed problem

$$
\begin{aligned}
-\left(p \phi_{\varepsilon}^{\prime}\right)^{\prime}+q \phi_{\varepsilon} & =\lambda_{\varepsilon} s \phi_{\varepsilon}, \quad 0<x<l+\varepsilon \\
\phi_{\varepsilon}(0) & =\phi_{\varepsilon}(l+\varepsilon)=0
\end{aligned}
$$

and rescaling it to the fixed interval $[0, l]$ :

$$
\begin{aligned}
-\left(\tilde{p} \tilde{\phi}_{\varepsilon}^{\prime}\right)^{\prime}+\left(\frac{l+\varepsilon}{l}\right)^{2} \tilde{q} \tilde{\phi}_{\varepsilon} & =\lambda_{\varepsilon}\left(\frac{l+\varepsilon}{l}\right)^{2} \tilde{s} \tilde{\phi}_{\varepsilon}, \quad 0<\tilde{x}<l, \\
\tilde{\phi}_{\varepsilon}(0) & =\tilde{\phi}_{\varepsilon}(l)=0 .
\end{aligned}
$$

Here $\tilde{\phi}_{\varepsilon}(\tilde{x})=\phi_{\varepsilon}((l+\varepsilon) \tilde{x} / l)$, and similar expressions hold for $\tilde{p}, \tilde{q}$, and $\tilde{s}$.

To conclude that

$$
\lambda_{\varepsilon}=\lambda+\varepsilon \lambda^{(1)}+o(\varepsilon) \text { and } \phi_{\varepsilon}=\phi+\varepsilon \phi^{(1)}+o(\varepsilon)
$$

it is sufficient that the eigenvalue $\lambda$ be stable with respect to the perturbation (in the sense that an isolated eigenvalue remains isolated) and that the perturbed differential operator converge to the original in an appropriate sense (that of generalized strong convergence) [ 3 , Chapter VIII]. The first condition is immediately verified since the differential operators are regular for all $\varepsilon$ sufficiently small and have only isolated, simple eigenvalues. The second condition can be verified if $p, q$, and $s$ are 
sufficiently smooth-it is enough to have $p \in C^{2}[0,1]$ and $q, s \in C^{1}[0,1]$ - using the general theory in $[3$, loc. cit.]. The details are not central to this paper and are omitted.

Expressions for $\partial \lambda_{i} / \partial x_{i}$ and $\partial \lambda_{i} / \partial x_{i+1}$ (the only nontrivial partial derivatives of $\lambda_{i}$ ) can be obtained using perturbation expansions. Consider

$$
\begin{gathered}
L \phi_{\mu \varepsilon}=-\left(p \phi_{\mu \varepsilon}^{\prime}\right)^{\prime}+q \phi_{\mu \varepsilon}=\lambda s \phi_{\mu \varepsilon}, \quad x_{i}+\mu<x<x_{i+1}+\varepsilon, \\
\phi_{\mu \varepsilon}\left(x_{i}+\mu\right)=\phi_{\mu \varepsilon}\left(x_{i+1}+\varepsilon\right)=0 .
\end{gathered}
$$

Expand the boundary conditions about $x_{i}$ and $x_{i+1}$ to get

$$
0=\phi_{\mu \varepsilon}\left(x_{i}+\mu\right)=\phi_{\mu \varepsilon}\left(x_{i}\right)+\phi_{\mu \varepsilon}^{\prime}\left(x_{i}\right) \mu+\cdots
$$

and

$$
0=\phi_{\mu \varepsilon}\left(x_{i+1}+\varepsilon\right)=\phi_{\mu \varepsilon}\left(x_{i+1}\right)+\phi_{\mu \varepsilon}^{\prime}\left(x_{i+1}\right) \varepsilon+\cdots .
$$

Substituting for $\lambda_{\mu \varepsilon}$ and $\phi_{\mu \varepsilon}$ the expansions

$$
\lambda_{\mu \varepsilon}=\lambda+\lambda_{10} \mu+\lambda_{01} \varepsilon+\cdots \quad \text { and } \phi_{\mu \varepsilon}=\phi+\phi_{10} \mu+\phi_{01} \varepsilon+\cdots
$$

and gathering the terms of order $1, \mu$, and $\varepsilon$, we get

$$
\begin{aligned}
& (L-\lambda s) \phi=0, \quad \phi\left(x_{i}\right)=\phi\left(x_{i+1}\right)=0 ; \\
& (L-\lambda s) \phi_{10}=\lambda_{10} s \phi, \quad \phi_{10}\left(x_{i}\right)+\phi^{\prime}\left(x_{i}\right)=\phi_{10}\left(x_{i+1}\right)=0 ; \quad \text { and } \\
& (L-\lambda s) \phi_{01}=\lambda_{01} s \phi, \quad \phi_{01}\left(x_{i}\right)=\phi_{01}\left(x_{i+1}\right)+\phi^{\prime}\left(x_{i+1}\right)=0 .
\end{aligned}
$$

The first equation above is just the original unperturbed eigenvalue problem for $\lambda$. From the second equation we obtain (using integration by parts)

$$
\begin{aligned}
\lambda_{10}(\phi, \phi)_{s, i} & =\left((L-\lambda s) \phi_{10}, \phi\right)_{i}=\left(\phi_{10},(L-\lambda s) \phi\right)+\left.p\left(\phi_{10} \phi^{\prime}+\phi \phi_{10}^{\prime}\right)\right|_{x_{i}} ^{x_{i+1}} \\
& =-p\left(x_{i}\right) \phi_{10}\left(x_{i}\right) \phi^{\prime}\left(x_{i}\right)=p\left(x_{i}\right) \phi^{\prime}\left(x_{i}\right)^{2} .
\end{aligned}
$$

Therefore $\lambda_{10}=p\left(x_{i}\right) \phi^{\prime}\left(x_{i}\right)^{2} /(\phi, \phi)_{s, i}$. The term $\lambda_{01}$ is handled similarly, and we get

$$
\frac{\partial \lambda_{i}}{\partial x_{i}}=p\left(x_{i}\right) \phi_{i}^{\prime}\left(x_{i}\right)^{2} \text { and } \frac{\partial \lambda_{i}}{\partial x_{i+1}}=-p\left(x_{i+1}\right) \phi_{i}^{\prime}\left(x_{i+1}\right)^{2},
$$

where $\phi_{i}$ is the normalized $\left(\left(\phi_{i}, \phi_{i}\right)_{s, i}=1\right)$ eigenfunction corresponding to the minimum eigenvalue $\lambda_{i}$ on $\left[x_{i}, x_{i+1}\right]$. Thus the Jacobian matrix $F^{\prime}(\mathbf{x})$ has the form

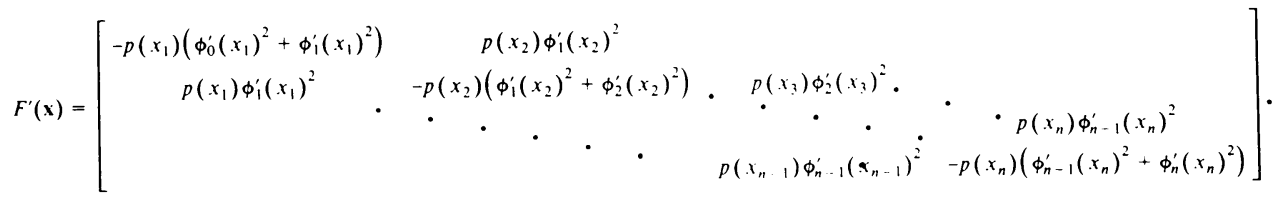

To evaluate $F^{\prime}(\mathbf{x})$, then, one needs information about the eigenfunctions $\phi_{0}, \ldots, \phi_{n}$ corresponding to the first eigenvalues of the subproblems. Depending upon what method is used to approximate $\lambda_{0}, \ldots, \lambda_{n}$, approximations to $\phi_{0}, \ldots, \phi_{n}$ should be available (at some additional expense). The use of asymptotic expressions for these eigenfunctions, as discussed below, obviates this additional computational effort. 
As $n$ increases, the subintervals $\left[x_{i}, x_{i+1}\right]$ become smaller and the subeigenvalues $\lambda_{0}, \ldots, \lambda_{n}$ become larger. Thus, in the eigenvalue equation

$$
\begin{gathered}
-\left(p \phi_{i}^{\prime}\right)^{\prime}+q \phi_{i}=\lambda_{i} s \phi_{i}, \quad x_{i}<x<x_{i+1}, \\
\phi_{i}\left(x_{i}\right)=\phi_{i}\left(x_{i+1}\right)=0
\end{gathered}
$$

the term $\lambda_{i} s \phi_{i}$ dominates the term $q \phi_{i}$ and the WKB (or Liouville-Green) approximation becomes effective (see [4, Section 7.1.3]). This can be obtained as follows. In the equation

$$
-\left(p u^{\prime}\right)^{\prime}+q u=\lambda s u
$$

where $u=u(x)$, transform the dependent and independent variables according to

$$
z=\int^{x} \sqrt{\frac{s}{p}} \quad \text { and } \quad v(z)=\psi(x) u(x)
$$

where $\psi(x)=(s p)^{1 / 4}(x)$. This produces

$$
v^{\prime \prime}+\lambda v-\frac{1}{s}\left[\psi \cdot\left(\frac{p \psi^{\prime}}{\psi^{2}}\right)^{\prime}+q\right] v=0 \quad \text { or } \quad v^{\prime \prime}+(\lambda+\delta) v=0
$$

where $\delta$ is independent of $\lambda$ and is a complicated expression in $s, p$, and $q$ and their derivatives up to second order. To leading order (for $\lambda$ large), the solutions of the transformed equation are $\sin (\sqrt{\lambda} z)$ and $\cos (\sqrt{\lambda} z)$, and we get the asymptotic formula

$$
\phi_{i}(x) \sim \frac{1}{(p s)^{1 / 4}(x)} \begin{cases}\sin \left[\lambda_{i}^{1 / 2} \int_{x_{i}}^{x}\left(\frac{s}{p}\right)^{1 / 2}\right], & x \text { near } x_{i} \\ \sin \left[\lambda_{i}^{1 / 2} \int_{x}^{x_{i+1}}\left(\frac{s}{p}\right)^{1 / 2}\right], & x \text { near } x_{i+1} .\end{cases}
$$

Some calculations give

$$
\begin{gathered}
\left(\phi_{i}, \phi_{i}\right)_{s, i} \sim \frac{1}{2} \int_{x_{i}}^{x_{i+1}}\left(\frac{s}{p}\right)^{1 / 2}-\frac{1}{4 \lambda_{i}^{1 / 2}} \sin \left[2 \lambda_{i}^{1 / 2} \int_{x_{i}}^{x_{i+1}}\left(\frac{s}{p}\right)^{1 / 2}\right] \\
p\left(x_{i}\right) \phi_{i}^{\prime}\left(x_{i}\right)^{2} \sim\left(\frac{s}{p}\right)^{1 / 2}\left(x_{i}\right) \lambda_{i} \text { and } p\left(x_{i+1}\right) \phi_{i}^{\prime}\left(x_{i+1}\right)^{2} \sim\left(\frac{s}{p}\right)^{1 / 2}\left(x_{i+1}\right) \lambda_{i}
\end{gathered}
$$

Because of the asymptotic relation $\lambda_{i}^{1 / 2} \int_{x_{i}}^{x_{i+1}}(s / p)^{1 / 2} \sim \pi$, we can take as a first, approximation

$$
\frac{\partial \lambda_{i}}{\partial x_{i}} \sim \frac{2}{\pi}\left(\frac{s}{p}\right)^{1 / 2}\left(x_{i}\right) \lambda_{i}^{(3 / 2)} \text { and } \frac{\partial \lambda_{i}}{\partial x_{i+1}} \sim \frac{-2}{\pi}\left(\frac{s}{p}\right)^{1 / 2}\left(x_{i+1}\right) \lambda_{i}^{(3 / 2)}
$$

Actually, using the more complete expressions (3.1) (accurately approximating the integrals) produced negligible improvement in the convergence of the method for the problems tested. 
Thus we have the approximate Jacobian $A(\mathbf{x})$ given by

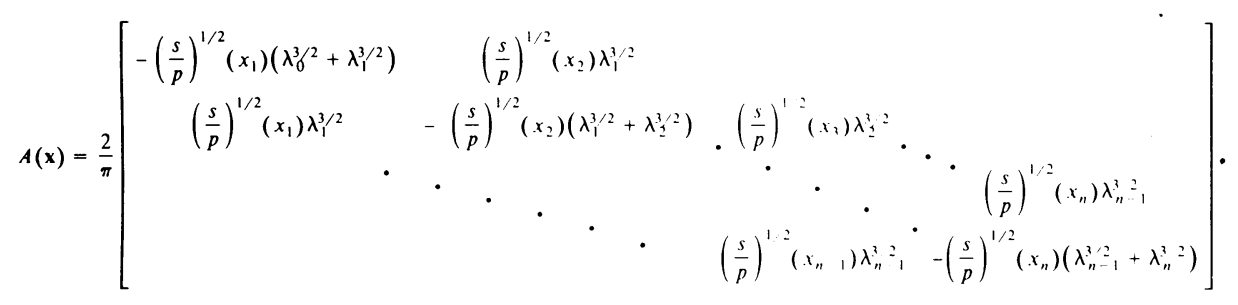

Now for all $\mathbf{x}$ in $D, F^{\prime}(\mathbf{x})$ and $A(\mathbf{x})$ are nonsingular; in fact, $F^{\prime}(\mathbf{x})^{T}$ and $A(\mathbf{x})^{T}$ are irreducible diagonally dominant [6]. Furthermore, it is a consequence of the expansion

$$
\phi_{\mu \varepsilon}=\phi+\mu \phi_{10}+\varepsilon \phi_{01}+o(\varepsilon)+o(\mu)
$$

and the continuity of $s, p$, and $\lambda$, that $F^{\prime}(\mathbf{x})$ and $A(\mathbf{x})$ are continuous functions of $\mathbf{x}$ on $D$. So the Newton iteration

$$
\begin{aligned}
& \mathbf{x}^{(0)} \text { given } \\
& \mathbf{x}^{(k+1)}=\mathbf{x}^{(k)}-F^{\prime}\left(\mathbf{x}^{(k)}\right)^{-1} F\left(\mathbf{x}^{(k)}\right), \quad k=0,1, \ldots
\end{aligned}
$$

and the approximate Newton iteration

$$
\begin{aligned}
& \mathbf{x}^{(0)} \text { given } \\
& \mathbf{x}^{(k+1)}=\mathbf{x}^{(k)}-A\left(\mathbf{x}^{(k)}\right)^{-1} F\left(\mathbf{x}^{(k)}\right), \quad k=0,1, \ldots
\end{aligned}
$$

both have the unique fixed point $\xi=\left(\xi_{1}, \ldots, \xi_{n}\right)^{T}$ (the zeros of $\Phi_{n+1}$ ) in $D$. It follows that $\xi$ is a point of attraction for the Newton iteration (once one gets sufficiently close) as it will be for the approximate Newton iteration if $\left\|A(\xi)-F^{\prime}(\xi)\right\|$ is sufficiently small (cf. [5]). The approximate Newton scheme was implemented with the initial points equally spaced, and in no tested problem did it fail to converge.

4. Numerical Results. There is a good deal of freedom in how one chooses to make use of the material in sections two and three. The basic block upon which any procedure must be built is a subroutine to approximate, accurately and efficiently, the minimum eigenvalue of a regular Sturm-Liouville problem. This routine will be called repeatedly to compute the subeigenvalues $\lambda_{0}, \ldots, \lambda_{n}$ while the approximate Newton iteration attempts to equilibrate these values.

The implementation used to generate the results reported in this section employed finite differences and extrapolation. Standard central differences were used to discretize the differential eigenvalue problem with $N$ equally spaced interior mesh points for $N+1=4,8,16,32,64$. The minimum eigenvalues of similar, symmetric, tridiagonal matrices were then found using a rational $Q R$ routine from the IMSL package. Richardson extrapolation was used to produce a high-accuracy approximation. The computations were done on a CDC 6600, the single floating-point 
precision of which is about 14 decimal digits. For test problems, the approximate eigenvalue typically contained 11 or 12 significant digits, and further extrapolation could not improve on this.

An approximate Newton method, as described in Section 3, was used to equilibrate the subeigenvaluess $\lambda_{0}, \ldots, \lambda_{n}$. The initial guess was the vector of equally spaced points $x_{j}^{(0)}=j /(n+1), j=1, \ldots, n$. The iteration was continued to singleprecision machine- attainable accuracy.

Actually there is no need to approximate $\lambda_{0}, \ldots, \lambda_{n}$ to high accuracy in the early stages of the iteration, and some efficiency can be gained by only computing up to $N+1=16$ or 32 , or so, when one can get away with it. Since a reliable bound of the current error is available in the form of the relative equilibration error $\left(\lambda_{\max }-\lambda_{\min }\right) / \lambda_{\min }$ and since quadratic convergence of the Newton iteration is expected, one can ask the subroutine to provide twice the accuracy of the current approximation at any stage.

\section{TABLE 1}

Relative error in approximation of zeros of $(n+1)$ st eigenfunction of Example (4.1)

\begin{tabular}{|c|c|c|c|}
\hline$n$ & Iteration & $\begin{array}{l}\text { Maximum Relative Error } \\
\text { in the } n \text { zeros }\end{array}$ & $\begin{array}{c}\text { Relative } \\
\text { Equilibration Error }\left(\frac{\lambda_{\max }{ }^{-\lambda} \min }{\lambda_{\min }}\right)\end{array}$ \\
\hline \multirow[t]{5}{*}{4} & 0 & $1.4(-1)$ & $1.0(0)$ \\
\hline & 1 & $1.3(-2)$ & $1.3(-1)$ \\
\hline & 2 & $5.8(-4)$ & $4.2(-3)$ \\
\hline & 3 & $4.1(-7)$ & $5.5(-6)$ \\
\hline & 4 & $1.8(-12)$ & $1.5(-11)$ \\
\hline \multirow[t]{6}{*}{8} & 0 & $1.5(-1)$ & $1.2(0)$ \\
\hline & 1 & $1.4(-2)$ & $2.0(-1)$ \\
\hline & 2 & $7.9(-4)$ & $1.1(-2)$ \\
\hline & 3 & $3.6(-6)$ & $6.5(-5)$ \\
\hline & 4 & $1.3(-10)$ & $2.5(-9)$ \\
\hline & 5 & $3.9(-13)$ & $7.3(-12)$ \\
\hline \multirow[t]{6}{*}{12} & 0 & $1.5(-1)$ & $1.2(0)$ \\
\hline & 1 & $1.5(-2)$ & $2.3(-1)$ \\
\hline & 2 & $8.4(-4)$ & $1.6(-2)$ \\
\hline & 3 & $5.2(-6)$ & $1.4(-4)$ \\
\hline & 4 & $4.5(-10)$ & $1.2(-9)$ \\
\hline & 5 & $7.8(-13)$ & $1.1(-11)$ \\
\hline
\end{tabular}


TABLE 2

Relative error in approximation of $(n+1)$ st eigenvalue in Example (4.2)

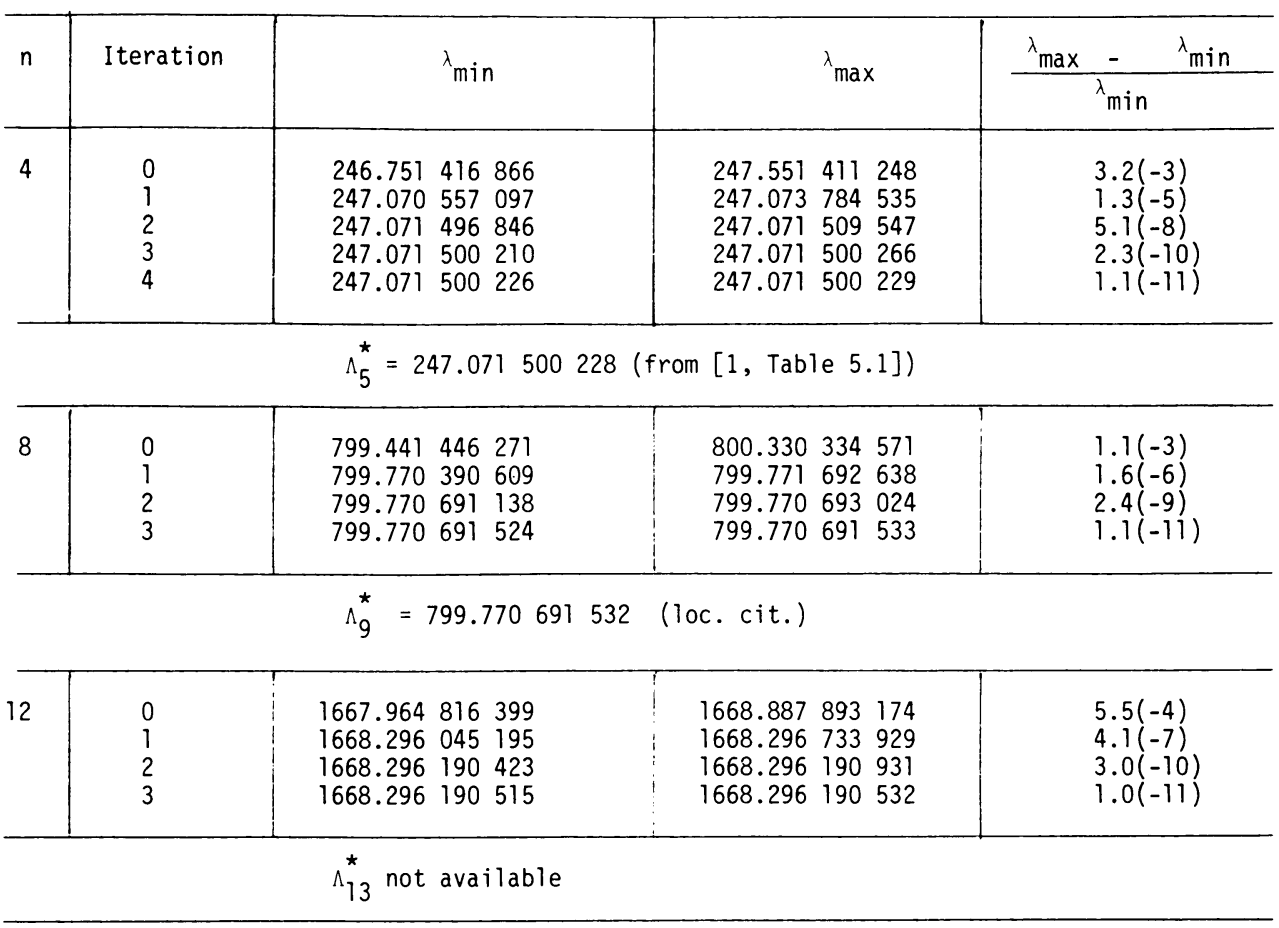

Numerical results are reported for three sample problems:

$$
\begin{gathered}
-\left(\frac{1}{\cosh x} \phi^{\prime}\right)^{\prime}=\Lambda \cosh x \phi, \quad 0<x<1, \\
\phi(0)=\phi(1)=0, \quad 0<x<1, \\
-\phi^{\prime \prime}+x^{2} \phi=\Lambda \phi, \quad 0, \\
\phi(0)=\phi(1)=0
\end{gathered}
$$

and

$$
\begin{gathered}
-\phi^{\prime \prime}+x|x| \phi=\Lambda \phi, \quad-1<x<1, \\
\phi(-1)=\phi(1)=0 .
\end{gathered}
$$

Example (4.1) has eigenvalues and eigenfunctions given by

$$
\Lambda_{n}=\left(\frac{n \pi}{\sinh 1}\right)^{2} \text { and } \Phi_{n}(x)=\sin \left(\frac{n \pi}{\sinh 1} \sinh x\right), \quad n=1,2, \ldots
$$

The true zeros of the $(n+1)$ st eigenfunction are then

$$
\xi_{j}=\sinh ^{-1}\left(\frac{j}{n+1} \sinh 1\right), \quad j=1, \ldots, n .
$$

This also happens to be an example where the WKB approximation is exact. Results are reported in Table 1. The anticipated quadratic convergence of Newton's method is observed; the table also shows the error in the zeros to be of the same order as the 
TABLE 3

Relative error in approximation of $(n+1)$ st eigenvalue of Example (4.3)

\begin{tabular}{|c|c|c|c|c|}
\hline$n$ & Iteration & $\lambda_{\min }$ & $\lambda_{\max }$ & $\frac{\lambda_{\max }-\lambda_{\min }}{\lambda_{\min }}$ \\
\hline 4 & $\begin{array}{l}0 \\
1 \\
2 \\
3 \\
4 \\
5\end{array}$ & 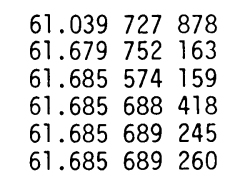 & $\begin{array}{lll}62.330 & 183 & 164 \\
61.694 & 424 & 745 \\
61.685 & 799 & 629 \\
61.685 & 590 & 353 \\
61.685 & 689 & 274 \\
61.685 & 689 & 261\end{array}$ & $\begin{array}{l}2.1(-2) \\
2.4(-4) \\
3.7(-6) \\
3.1(-8) \\
4.6(-10) \\
9.0(-12)\end{array}$ \\
\hline \multicolumn{5}{|c|}{$\Lambda_{5}^{\star}=61.6856893$ (from [1, Table 5.2]) } \\
\hline 8 & $\begin{array}{l}0 \\
1 \\
2 \\
3 \\
4\end{array}$ & $\begin{array}{l}199.067743737 \\
199.857703989 \\
199.859697451 \\
199.859724772 \\
199.859724824\end{array}$ & $\begin{array}{lll}200.651 & 217 & 589 \\
199.864 & 748 & 970 \\
199.859 & 751 & 850 \\
199.859 & 724 & 954 \\
199.859 & 724 & 828\end{array}$ & $\begin{array}{l}8.0(-3) \\
3.5(-5) \\
2.7(-7) \\
9.1(-10) \\
1.9(-11)\end{array}$ \\
\hline \multicolumn{5}{|c|}{$\Lambda_{9}^{\star}=199.8597250$ (loc. cit.) } \\
\hline 12 & $\begin{array}{l}0 \\
1 \\
2 \\
3 \\
4\end{array}$ & 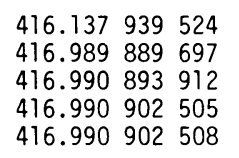 & 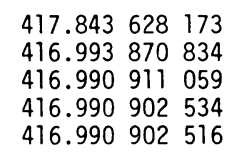 & $\begin{array}{l}4.1(-3) \\
9.5(-6) \\
4.1(-8) \\
6.9(-11) \\
1.5(-11)\end{array}$ \\
\hline \multicolumn{5}{|c|}{$\Lambda_{13}^{*}=416.9909052$ (loc. cit.) } \\
\hline
\end{tabular}

equilibration error-as one would expect since $F^{\prime}(\xi)$ is nonsingular. We are able to approximate these points to within 2 digits of full single-precision machine accuracy without using any extended precision or ever actually approximating $\Phi_{n+1}$.

Example (4.2) is taken from [1], where 12-digit approximations to the first several eigenvalues are given. For this example, the true eigenfunctions are parabolic cylinder functions; the WKB approximation is certainly not exact. The approximate Newton method, then, is expected to converge linearly with the number of digits gained at each stage depending on the accuracy of the approximation to the Jacobian. The results are reported in Table 2. The initial guess of equally-spaced points is quite good in this case, giving 2 to 3 significant digits to begin. The convergence is linear but picks up about 3 decimal digits per iteration. The final brackets contain the correct eigenvalues in both cases $\Lambda_{5}$ and $\Lambda_{9}$. Though this is not guaranteed, because of the random effect of error at this maximum attainable accuracy, the final values strongly suggest that the first 10 digits are correct.

The final example, (4.3), is also taken from [1]. The eigenfunctions for this problem are in $C^{4}[-1,1]$ with piecewise-continuous 5 th derivative. The calculations of [1] use the Rayleigh-Ritz method with a basis of trigonometric functions. This gives exponential convergence if the eigenfunctions are analytic but here gives a convergence rate of the order $O\left(N^{-6}\right)$ for an $N$-dimensional subspace with $N=16$. 
For this example, 10 decimal digits are given in [1], about 8 or 9 of which appear to be correct. Our results for this problem are reported in Table 3. The approximate Newton algorithm again converged in 4 or 5 iterations, and about 10 or 11 digits of accuracy in $\Lambda_{5}, \Lambda_{9}$, and $\Lambda_{13}$ appear to be obtained.

5. Conclusion. The results of the previous section are typical of those over a wide range of problems tested. The approximate Newton scheme generally took about 4 to 5 iterations to converge to full accuracy (once it had a digit or so). In no case did the method fail to converge. Final accuracy in the zeros and eigenvalue was always of the order of 11 or 12 significant digits.

The basic approach seems to provide an effective scheme for approximating to high accuracy the eigenvalues and zeros of selected eigenfunctions for such regular Sturm-Liouville problems, especially in the "intermediate" range between where Rayleigh-Ritz and finite differences are satisfactory and where asymptotic formulas do well enough. The unly limitation on attainable accuracy is the precision with which one is able to approximate the minimum eigenvalue of a regular Sturm-Liouville problem. This is, in general, an easier problem (for which to attain high accuracy) than approximating $\Phi_{n+1}$ or $\Lambda_{n+1}$ directly: with the Rayleigh-Ritz method, for example, the degree of approximation to $\Lambda_{n+1}$ is roughly twice that to $\Phi_{n+1}$ (due to the fact that $\Lambda_{n+1}$ is a quadratic function of $\Phi_{n+1}$ ), while with finite-difference methods, extrapolation can be employed to provide a much more accurate approximation to $\Lambda_{n+1}$ than the best available approximation to $\Phi_{n+1}$. Also, since the method is iterative, good estimates of the accuracy in the approximate roots and eigenvalues can be inferred by simply observing the converging terms of the sequence.

The procedure is not inexpensive, requiring the calculation of $n+1$ minimum eigenvalues $\lambda_{0}, \ldots, \lambda_{n}$, for each iteration; but as observed, one can get by with lower accuracy initially or stop the iteration when the relative equilibration error gets below an acceptable tolerance. The alternatives are not inexpensive either, for that matter, and involve algebraic eigenvalue problems of larger and larger size as $n$ increases or as one seeks more accuracy. With this equilibration approach, some aspects of the computations even improve with $n$ : for one thing, the size of the problem does not grow (the largest array used has dimension 63, independent of $n$ ), and for another thing, as the subintervals get smaller, the coefficient of the differential operator appear more constant, improving the accuracy of the subeigenvalue approximations, and $\lambda_{0}, \ldots, \lambda_{n}$ get larger, improving the accuracy of the WKB approximation and rate of convergence of the approximate Newton iteration.

The programming effort for the approach is small, and as implemented here, the method does not require any extended precision. Also, the method had the advantage that it is general; it requires no particular information about coefficients of eigenfunctions.

Acknowledgement. The author is grateful to Rich Haberman and Andreas Griewank (both of Southern Methodist University) for helpful discussions and to the referee for his suggestions. 
Added in Proof. The variational characterization (2.1) can be seen as a consequence of the fact that the Green's function associated with the differential operator $L$ is totally positive [A. A. Melkman \& C. A. Micchelli, "Spline spaces are optimal for $L^{2}$-width," Illinois J. Math., v. 22, 1978, pp. 541-564.]. The author is grateful to Charles Micchelli for this communication.

Department of Mathematics

Southern Methodist University

Dallas, Texas 75275

1. G. BIRKHOFF \& G. FIX, "Accurate eigenvalue computations for elliptic problems," in Numerical Solution of Field Problems in Continuum Physics (G. Birkhoff and R. S. Varga, eds.), Amer. Math. Soc., Providence, 1970, pp. 111-151.

2. E. A. Coddington \& N. Levinson, Theory of Ordinary Differential Equations, McGraw-Hill, New York, 1955.

3. T. Kato, Perturbation Theory for Linear Operators, Springer-Verlag, New York, 1966.

4. A. H. NA YFeH, Perturbation Methods, Wiley-Interscience, New York, 1973.

5. J. M. ORTEGA \& W. C. RHEINBOLDT, Iterative Solution of Nonlinear Equations in Several Variables, Academic Press, New York, 1970.

6. R. S. VARGA, Matrix Iterative Analysis, Prentice-Hall, Englewood Cliffs, N.J., 1962.

7. H. F. WeInberger, Variational Methods for Eigenvalue Approximation, SIAM, Philadelphia, Pa., 1974. 